\title{
4 Los factores verbales desde una perspectiva sincrónica: estudio de cuestionarios
}

\subsection{Introducción}

El principal objetivo de este capítulo es el de investigar la relación que existe entre la telicidad, la afectación y el MDO en español. Como han apuntado los capítulos previos, la relación de estos dos factores con el marcado representa una cuestión cuyo análisis hasta el momento ha sido escaso y carente de datos empíricos sobre los que sustentar las afirmaciones que se han realizado.

Para afrontar este cometido, tanto este capítulo como el capítulo 5 se han orientado hacia la investigación empírica del impacto que la telicidad y la afectación ejercen sobre el MDO. De forma paralela a los capítulos 2 y 3, el presente capítulo aborda la cuestión desde un punto de vista sincrónico, mientras que en el capítulo 5 se adopta un enfoque de corte diacrónico. La finalidad es obtener suficientes datos desde ambas perspectivas que, de ser coherentes, sean capaces de ofrecer una visión panorámica del efecto que los factores investigados han ejercido y ejercen sobre el MDO en español.

El estudio sincrónico que se presenta a lo largo de las siguientes páginas ha consistido en la realización de un cuestionario de elección forzada, acompañado del correspondiente análisis de sus resultados. La investigación sincrónica acerca del marcado preposicional ha puesto al descubierto la relevancia del factor de la afectación en la explicación del fenómeno, mientras que la telicidad no ha mostrado en el experimento un impacto que corrobore las teorías propuestas por la bibliografía previa.

El presente capítulo consta de cinco partes, sin tener en cuenta este breve apartado introductorio. El apartado 4.2 presenta las hipótesis sobre las que se construye este primer estudio de corte sincrónico, establecidas a partir de las ideas obtenidas en los capítulos previos. A continuación, en el 4.3 se describe la metodología que se ha empleado y la justificación de esta. En el apartado 4.4 se exponen los resultados obtenidos en el cuestionario y en el 4.5 estos son analizados y comentados. Finalmente, el apartado 4.6 recoge los aspectos y las ideas más destacadas que han surgido a lo largo del capítulo.

\subsection{Hipótesis}

Telicidad y afectación son dos nociones que, como se ha mostrado en los capítulos 2 y 3, han dado pie a numerosos estudios motivados desde muy diferen- 
tes puntos de vista y, por lo tanto, el uso que se ha hecho de ambos conceptos presenta una variación considerable. Por esta razón, es fundamental comenzar el planteamiento de este estudio empírico recordando la definición que se ha tomado en este caso para cada uno de ellos, a pesar de que ya se ha señalado a lo largo de los capítulos previos.

Para comenzar, se considera telicidad aquella propiedad del SV que implica la existencia de un punto final o culminación tras el cual el evento designado por el predicado finaliza o se completa (Verkuyl 1972; entre otros; Dowty 1991; Tenny 1994; Krifka 1998). Esta definición es la que parece recabar más apoyos en la bibliografía, tal y como se ha podido observar en el apartado 2.2.6.1. Por un lado, los trabajos que circunscriben la telicidad al núcleo verbal plantean evidentes problemas para establecer el valor aspectual de predicados como el de (82b). Ejemplos como este, en los que un mismo verbo puede aparecer con diferentes estructuras argumentales, son la razón para no reducir únicamente al verbo el ámbito en el que se genera la telicidad e incluir aquellos constituyentes del predicado que contribuyen a establecer el valor aspectual (véase Marín 1999 para un análisis más detallado).

(82) Correr con diferente estructura argumental
a. María corre.
(un argumento; verbo de actividad)
b. María corre un kilómetro. (dos argumentos; verbo de realización)

Por otro lado, se ha determinado no incluir en la computación de la telicidad elementos externos al SV, tales como el sujeto o el aspecto gramatical, puesto que los enfoques que defienden su incorporación reconocen que el impacto de estos se produce de manera más indirecta. En ocasiones no queda del todo claro si es el propio sujeto el que realmente mide el evento, o si lo que produce el aspecto gramatical es un cambio del valor aspectual o directamente una coacción para que el verbo adopte otra de sus posibles acepciones. Obsérvense los ejemplos de (83). El primero de dichos ejemplos es mencionado por Krifka $(1998,29)$ para explicar la relación entre el argumento externo y la delimitación del evento acudiendo al concepto de tema incremental. La idea es que la correspondencia entre las partes de un argumento y las partes del evento no tiene por qué producirse solo en el caso del argumento interno, sino que puede darse también entre el argumento externo y el evento, y, según Krifka, es lo que ocurriría en (83a'). La cuestión fundamental es que se trate de un argumento divisible en partes relevantes. Según el autor, mientras que en (83a) es el argumento interno el que delimita el evento (mediante la aportación implícita de una vía y un límite representados por la anchura del río y la orilla opuesta), en (83a') parece que el argumento que cobra mayor peso como «medidor» del evento sería el sujeto, es decir, la medición del 
evento se desprendería de la asignación de las distintas partes de el ejército (los soldados) a las correspondientes partes del evento. No obstante, esta idea puede resultar problemática, pues el predicado cruzar el río es télico tanto en (83a) como en (83a'), lo que dificulta determinar que sea el sujeto quien tiene la capacidad de medir el evento en el segundo de los casos.

(83) Elementos externos al SV y la construcción de la telicidad

a. El soldado cruzó el río.

a'. El ejército cruzó el río.

b. El anfitrión conoció a su invitado.

b'. El anfitrión conocía a su invitado.

En cuanto a el contraste entre (83b) y (83b'), del primero de los casos parece obtenerse una lectura télica, mientras que, en el segundo, la mayoría de los hablantes coincidirían en señalar una lectura atélica. Si bien es cierto que lo único que varía de un ejemplo a otro es el aspecto gramatical de conocer, este cambio lo que parece coaccionar realmente es el significado que el verbo adopta en cada caso y, no tanto, el valor aspectual del predicado. Así, mientras que en (83b), conocer se interpreta en el sentido de 'reconocer' o 'tener contacto por vez primera', en (83b') el sentido parece ser el de 'tener conciencia de'. En conclusión, como no queda claro el efecto del sujeto sobre la telicidad y el impacto del aspecto gramatical parece ser tan solo indirecto, la determinación que se ha tomado es la de reducir el ámbito de configuración de la telicidad tan solo al SV.

En lo que respecta a la evaluación de la telicidad de los predicados, la prueba elegida ha sido la utilizada tradicionalmente, establecida por el propio Vendler (1957). Dicha prueba es la que se muestra en (84) y consiste en la adición de un adjunto al predicado, introducido por la preposición en, que establece un límite explícito en el evento. Como se observa en (84a), aquellos predicados que admiten dicho SP con función delimitadora son considerados télicos, mientras que aquellos que no lo admiten, como es el caso de (84b), son catalogados como atélicos.

(84) Prueba de telicidad

a. María corre un kilómetro en cinco minutos.

b. \#María corre por el parque en cinco minutos.

En lo que se refiere a la noción de afectación, esta es quizá la que mayor divergencia de usos ha experimentado. El capítulo 2 señalaba la existencia de dos grandes enfoques en los que se podían inscribir la mayoría de los trabajos acerca del tema: el enfoque espacio-temporal, basado en la idea de cambio, y la perspectiva basada en el concepto de transmisión de fuerza. 
Como ya se adelantaba, este trabajo ha optado por el segundo enfoque, es decir, el basado en la transmisión de fuerza, debido a que permite explicar algunos casos que quedaban excluidos del ámbito más restringido del enfoque espacio-temporal. Consecuentemente, la definición de afectación que se maneja de aquí en adelante es aquella que considera que un objeto afectado es todo aquel que participa en un evento y es receptor de una fuerza (Jackendoff 1990; Croft 1991). Esta definición ha demostrado ser útil, no solo para explicar el fenómeno del MDO, como muestra este trabajo; sino también para explicar otros fenómenos relacionados con la afectación, como el comportamiento de las estructuras causativas y las resultativas (Rappaport-Hovav/Levin 2001, 785-790). De hecho, la intención de Jackendoff $(1990,125)$ cuando propone la prueba de (85) es precisamente el análisis de la causatividad.

(85) Prueba de afectación (Jackendoff 1990, 125)

$\left\{\begin{array}{c}\text { Lo que le ocurrió } \\ \text { Lo que x le hizo }\end{array}\right\}$ a SN fue...

Esta es la única prueba específica que se ha propuesto para probar la afectación de los objetos, tal como reconoce Beavers (2011, 339). Jackendoff, en un primer momento, realmente propone la prueba para evaluar la «condición de paciente» de los objetos; sin embargo, Rappaport-Hovav/Levin (2001, 787) señalan que dicha prueba lo que apunta ciertamente es la condición de «receptor de fuerza» de un argumento, por lo que resulta ideal para capturar la noción de afectado que se maneja bajo el enfoque adoptado en esta investigación. Así pues, los SN que pueden aparecer en el contexto que plantea la prueba sin provocar la anomalía de la estructura serán aquellos que puedan considerarse afectados en el sentido antes descrito. Como puede verse en (86), el objeto directo del primer ejemplo resulta afectado por la acción descrita por el verbo y pasa sin problema alguno la prueba; mientras que el objeto de (86b), que no resulta afectado por la acción de conocer, en el sentido de 'tener conciencia de', provoca la anomalía de la estructura resultante.

(86) Prueba de afectación

a. Luis destrozó el coche.

Lo que le ocurrió a al coche fue que Luis lo destrozó.

Lo que Luis le hizo al coche fue destrozarlo.

b. María conoce a sus vecinos.

\#Lo que les ocurre a sus vecinos es que María los conoce.

\#Lo que María les hizo a sus vecinos fue conocerlos. 
Una vez establecidas con claridad las definiciones y las pruebas relativas a cada uno de los dos factores estudiados, es posible formular con mayor claridad las hipótesis en las que se basa el estudio de cuestionarios realizado. Dichas hipótesis aparecen recogidas en (87) y están basadas en los datos aportados por los escasos estudios previos que fueron presentados en el capítulo 2.

\section{(87) Hipótesis}

H1: Los objetos directos de predicados télicos presentan con mayor frecuencia MDO que los objetos directos de predicados atélicos.

H2: Los objetos directos afectados por la acción del verbo presentan con mayor frecuencia MDO que los objetos no afectados.

\subsection{Diseño del estudio}

La metodología escogida para comprobar las hipótesis presentadas en el apartado anterior ha sido el cuestionario de respuesta forzada, que se inscribe dentro del conjunto de pruebas basadas en los llamados juicios de gramaticalidad. Este tipo de pruebas implica la expresión, de forma indirecta, de la percepción de aceptabilidad de los hablantes, y permite recabar información sobre fenómenos que no ocurren de forma lo suficientemente frecuente en el uso espontáneo de la lengua como para que puedan ser investigados de otra forma. Lo que no arroja este tipo de cuestionarios es información sobre el tamaño de la diferencia entre condiciones (al menos de forma directa), es decir, el cuestionario nos permite saber que un determinado participante prefiere en un contexto concreto la opción con MDO a la opción sin marcado, pero no se puede saber si dicha preferencia era muy clara (la opción marcada le parecía totalmente aceptable, mientras la sin marcar le parecía agramatical) o si, por el contrario, se trataba de una preferencia leve (ambas opciones le parecían aceptables, pero la marcada ligeramente mejor que la sin marcar). No obstante, los cuestionarios de respuesta forzada, en concreto, están pensados para detectar diferencias cualitativas entre diferentes condiciones y son los que mayor fuerza explicativa tienen, estadísticamente hablando, dentro de las pruebas basadas en juicios de aceptabilidad, especialmente en el caso de efectos pequeños (Schütze/Sprouse 2014, 32). Precisamente, por esta razón es por la que se ha optado por la metodología descrita, puesto que el objeto de investigación es el impacto de dos rasgos que, de actuar, lo hacen por detrás de otra serie de factores más relevantes y, por lo tanto, es necesaria la utilización de una herramienta que sea capaz de capturar diferencias mínimas de variación.

El cuestionario utilizado para este estudio está basado en un diseño factorial de 2 x2 que incluye, como variables independientes, los factores de telicidad y 
afectación y, como variable dependiente, la presencia/ausencia de MDO. El cruce de factores que posibilita este diseño permite examinar tanto el efecto aislado que la telicidad y la afectación puedan ejercer sobre el MDO, como la interacción entre ambas variables independientes, en caso de que se produzca. Dicho cruce factorial da lugar a la creación de cuatro parejas de rasgos: [+afectado, +télico], [+afectado, -télico], [-afectado, -télico] y [-afectado, +télico]. Para representar cada pareja se han elegido ocho verbos con los que se han construido ocho oraciones diferentes que responden a los rasgos indicados para cada grupo. Las oraciones se han incluido en los diferentes conjuntos tras ser sometidas a las pruebas de telicidad y afectación mencionadas en el apartado anterior. La Tabla 4.1 recoge los verbos a partir de los que se han creado las oraciones del cuestionario.

Tabla 4.1: Verbos utilizados en el cuestionario.

\begin{tabular}{|c|c|c|c|c|c|}
\hline & \multirow[b]{3}{*}{ + télico } & \multicolumn{4}{|c|}{ AFECTACIÓN } \\
\hline & & \multicolumn{2}{|c|}{ + afectado } & \multicolumn{2}{|c|}{ - afectado } \\
\hline TELICIDAD & & $\begin{array}{l}\text { abatir } \\
\text { arrollar } \\
\text { derribar } \\
\text { destrozar }\end{array}$ & $\begin{array}{l}\text { eliminar } \\
\text { neutralizar } \\
\text { quemar } \\
\text { vencer }\end{array}$ & $\begin{array}{l}\text { conocer }^{19} \\
\text { encontrar } \\
\text { descartar } \\
\text { descubrir }\end{array}$ & $\begin{array}{l}\text { detectar } \\
\text { identificar } \\
\text { localizar } \\
\text { mencionar }\end{array}$ \\
\hline & - télico & $\begin{array}{l}\text { arrastrar } \\
\text { apalear } \\
\text { asediar } \\
\text { guiar }\end{array}$ & $\begin{array}{l}\text { maltratar } \\
\text { remolcar } \\
\text { transportar } \\
\text { zarandear }\end{array}$ & $\begin{array}{l}\text { admirar } \\
\text { amar } \\
\text { investigar } \\
\text { oír }\end{array}$ & $\begin{array}{l}\text { percibir } \\
\text { tener } \\
\text { ver } \\
\text { vigilar }\end{array}$ \\
\hline
\end{tabular}

El cuestionario consta de tres partes: (i) una breve introducción, en la que se explica el funcionamiento de la prueba y se restringe el acceso a personas mayores de edad, que fuesen hablantes nativos de cualquiera de las variedades de español de España; (ii) unas preguntas generales para conocer el contexto sociolingüístico de los participantes; y (iii) la batería de preguntas, en este caso oraciones, que constituye la tarea en sí. La labor de los participantes consistía en elegir en cada oración la opción que considerasen que «mejor les sonaba» de las dos que se ofrecían en cada caso, es decir, el objetivo era que eligiesen la que les resultase más natural o la que considerasen que ellos utilizarían. La batería de preguntas está formada por 48 elementos: 32 preguntas u oraciones de estudio, 11 de control y 5 de relleno (véase Anexo 1 para el listado completo de preguntas). Estos 48 elementos adquirían un orden aleatorio para cada uno de los participantes que

19 Con el significado de 'tener contacto por primera vez con una persona'.

20 Con el significado de 'darse cuenta de la presencia de'. 
accedieron al cuestionario, con el objetivo de evitar efectos derivados del hecho de que las preguntas mantengan siempre una posición determinada dentro de la estructura.

Las preguntas $\mathrm{u}$ oraciones de estudio son aquellas diseñadas con la intención de poner a prueba las hipótesis de las que parte el estudio y son las que responden al diseño descrito más arriba. En cuanto a las preguntas u oraciones de control, poseen una doble función. Por un lado, su cometido es el de comprobar que la atención de los participantes se mantiene a lo largo del cuestionario y estos no optan por estrategias de respuesta aleatoria para terminar la tarea. Hay dos tipos de preguntas de control: aquellas en las que el MDO es claramente obligatorio y aquellas en las que lo obligatorio es su ausencia. La obviedad de las soluciones a estas preguntas posibilita la detección de participantes cuya estrategia de respuesta se vuelve aleatoria en algún punto del cuestionario y, por lo tanto, permite la eliminación de estos, a fin de contemplar solo las respuestas de aquellos que prueban mantener la atención en la tarea hasta el final. Por otro lado, la segunda función de las preguntas de control consiste en evitar la uniformidad estructural de las preguntas del cuestionario, procurando de esta manera que los participantes no perciban como excesivamente monótono el avance de la tarea y esto pueda implicar el abandono. Esta última función la comparten con las oraciones o preguntas de relleno, en las que, a diferencia de las de control, se ofrecen dos opciones de respuesta totalmente aceptables. El sentido, por tanto, de incluir este último tipo de preguntas en vez de un mayor número de preguntas de control es el de evitar que los participantes puedan considerar que la respuesta es obvia en demasiadas preguntas y determinar que no merece la pena continuar con la tarea.

La estructura de los tres tipos de preguntas es la misma y consta de dos partes: una oración incompleta que carece de objeto directo y dos posibles opciones de respuesta candidatas a desempeñar dicha función. Las oraciones, como puede observarse en (88), están formadas por un sujeto, constituido siempre por un nombre propio; uno de los verbos escogidos para el estudio que aparecen en la Tabla 4.1, un espacio para el objeto directo y un adjunto. El orden en que aparecen las opciones de respuesta es aleatorio en cada pregunta, evitando así que el objeto directo con MDO aparezca siempre en la misma posición y pueda generar que los participantes desarrollen estrategias automatizadas de respuesta.

(88) Preguntas $\mathrm{u}$ oraciones de estudio

Irene oyó durante horas.

a) un niño

b) a un niño 
El verbo de las oraciones que conforman las preguntas se encuentra siempre en pretérito indefinido, en tercera persona del singular. En cuanto al adjunto, es el elemento que más variación presenta atendiendo al tipo de pregunta. Las oraciones de estudio siempre contienen como adjunto un SP que, o bien presenta de forma explícita el límite del evento, o bien establece su duración. La función de dicho sintagma, en estos casos, es la de reforzar la lectura télica o atélica del predicado que ya establece la propia semántica del verbo. Los adjuntos de las preguntas de control y de relleno no están sujetos a ningún tipo de restricción y abarcan desde SP a oraciones subordinadas de distinto tipo. Asimismo, las opciones de objeto directo que se ofrecen en este tipo de preguntas tampoco están sujetas a las restricciones establecidas para las preguntas de control, como muestran (89) y (90). Esta pretendida variación, tanto en la forma de los objetos directos, como de los adjuntos es con la que se procura evitar la sensación de monotonía, tal y como se ha expuesto entre las funciones de este tipo de preguntas.

(89) Pregunta u oración de control Sara denunció por hacer demasiado ruido.

a) a su vecina

b) su vecina

(90) Pregunta u oración de relleno Héctor observó merodeando por el barrio.

a) a varios policías

b) varios policías

La plataforma utilizada para la implementación del cuestionario ha sido Qualtrics. Se trata de una plataforma comercial para la gestión de la experiencia de los clientes, cuyo objetivo fundamental es permitir a las empresas conocer la opinión del público acerca de sus productos, a través de los diversos tipos de encuestas y cuestionarios que permite crear. Qualtrics se ha convertido en los últimos años en una herramienta de referencia en la investigación a través de Internet no solo en las Ciencias Humanas (Steen/Reijnierse/Burgers 2014, entre otros), sino también en el campo de las Ciencias Sociales (Boas/Christenson/Glick 2020, entre otros) y Naturales (Miller/Currie/O’Carroll 2019, entre otros). La variedad de opciones que esta herramienta ofrece a la hora de crear cuestionarios en línea ha sido el motivo por el que ha resultado la elegida para implementar el cuestionario para este estudio. En cuanto a la difusión de este, se llevó a cabo a través del envío directo del enlace de acceso a participantes potenciales, que, a su vez, en muchos casos colaboraron con el reenvío del enlace, expandiendo así la red de personas a las que este acabó llegando. 


\subsection{Resultados}

El acceso al cuestionario en línea se mantuvo abierto durante diez días. En este período 333 personas accedieron a él y, de ellas, 326 contestaron a la totalidad de las preguntas. Respecto a la revisión de las preguntas de control, se determinó eliminar a aquellos participantes que no alcanzasen el 80\% de respuestas correctas y, tras llevar a cabo el análisis de las respuestas a este tipo de preguntas, ningún participante de los que había rellenado al completo el cuestionario resultó excluido de la investigación. Por lo tanto, los resultados que se presentan a continuación se corresponden con una muestra de 326 hablantes nativos de español, mayores de edad, que participaron en la tarea de forma voluntaria.

El tratamiento de los datos se ha llevado a cabo utilizando Microsoft Excel, programa en el que se han elaborado todas las figuras que se recogen en este capítulo. En lo que respecta al análisis estadístico, se han utilizado R (Team 2019) y lme4 (Bates et al. 2015) para desarrollar un modelo de regresión logística de efectos mixtos (glmer) de la relación entre el MDO y los factores verbales que constituyen el objeto de estudio (telicidad y afectación), utilizando como efectos fijos dichos factores y, como efecto aleatorio, interceptos aleatorios ${ }^{21}$ para los participantes.

El Gráfico 4.1 representa las frecuencias relativas obtenidas en el cuestionario con respecto a la telicidad y en la Tabla 4.2 se recogen además los datos absolutos correspondientes. La parte gris oscura de cada una de las barras del gráfico representa las oraciones en las que se ha elegido la opción con MDO, mientras que la zona más clara, aquellas en las que se ha preferido la opción sin marcar. La cantidad que refleja cada barra se corresponde en cada caso con el porcentaje del total de oraciones atendiendo a la categoría en cuestión.

Los datos obtenidos del cuestionario indican que los participantes han elegido la opción con MDO, frente a la opción sin marcar, en un 83\% de los predicados atélicos. Por otro lado, en lo referente a los predicados télicos, la opción marcada ha sido elegida en un $80 \%$ de los casos. Esta diferencia mínima de tan solo tres puntos porcentuales ha resultado no ser significativa en el análisis estadístico (estimado: 0,036; $\mathrm{p}=0,599$ ) y, por ello, no se puede afirmar, según estos datos, que el factor de la telicidad ejerza un efecto significativo sobre la respuesta de los participantes.

21 Intercepto: Mat. (en un sistema de coordenadas) la distancia desde el origen hasta el punto en el cual una curva o una recta intersecciona con el eje. La función de establecer el intercepto como efecto aleatorio para los participantes, en este caso, es la de descartar los efectos que pudieran surgir derivados de la forma que cada participante tiene de evaluar las distintas preguntas. 




Gráfico 4.1: Frecuencia de respuestas atendiendo al MDO y a la telicidad.

Tabla 4.2: Frecuencia de respuestas atendiendo al MDO y a la telicidad.

\begin{tabular}{lllrr}
\hline & \multicolumn{2}{l}{ MDO } & \multicolumn{2}{c}{ NO MDO } \\
\hline atélico & $83 \%$ & $(4324 / 5216)$ & $17 \%$ & $(892 / 5216)$ \\
\hline télico & $80 \%$ & $(4180 / 5216)$ & $20 \%$ & $(1036 / 5216)$ \\
\hline
\end{tabular}

En lo que se refiere al impacto de la afectación, representado en el Gráfico 4.2, los participantes han elegido la opción marcada preposicionalmente en un 75\% de los casos con objetos no afectados, frente al $88 \%$ de los que contenían objetos afectados. La diferencia entre ambas categorías asciende a 13 puntos porcentuales que, respaldados por el análisis estadístico, en este caso sí suponen una diferencia significativa (estimado: 1,$351 ; \mathrm{p}<0,001$ ). Por lo tanto, se puede afirmar que el factor de la afectación determina de un modo significativo que los participantes del cuestionario elijan la opción con MDO en vez de la opción sin marcar. Los datos absolutos relativos a la afectación son los que se pueden observar en la Tabla 4.3.

Finalmente, el Gráfico 4.3 y su correspondiente tabla recogen los datos referidos a la interacción de ambos factores. Dichos datos muestran que, en contextos con objetos no afectados, se ha elegido la alternativa con MDO en un $75 \%$ de los casos tanto en los predicados atélicos, como en los télicos; y, en lo que concierne a los objetos afectados, los participantes han elegido la opción marcada en un $91 \%$ de los predicados atélicos, frente al $85 \%$ de los predicados télicos. Estos datos conducen al planteamiento de una interacción entre telicidad y afectación, avalada por el análisis estadístico (estimado: $-0,679 ; \mathrm{p}<0,001$ ), que implica que 


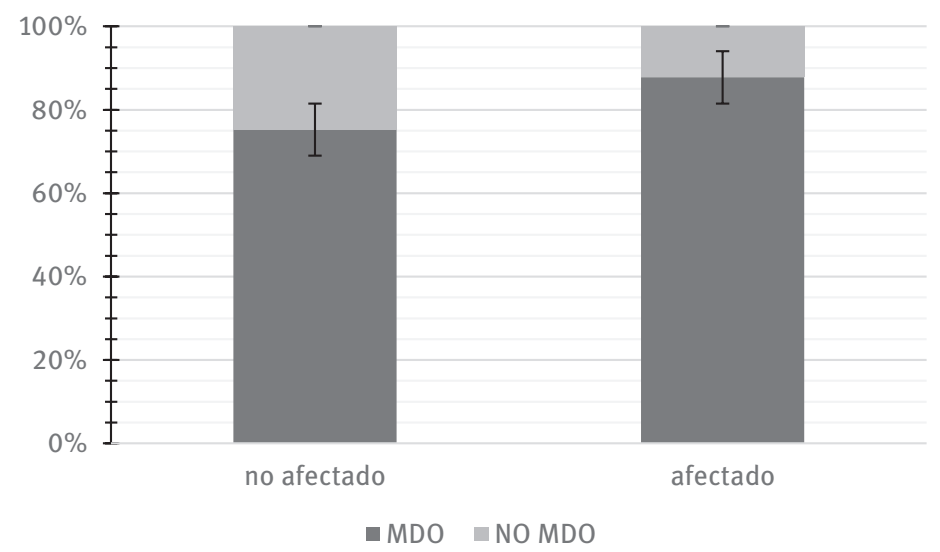

Gráfico 4.2: Frecuencia de respuestas atendiendo al MDO y a la afectación.

Tabla 4.3: Frecuencia de respuestas atendiendo al MDO y a la afectación.

\begin{tabular}{lllrr}
\hline & \multicolumn{2}{l}{ MDO } & \multicolumn{2}{c}{ NO MDO } \\
\hline no afectado & $75 \%$ & $(3925 / 5216)$ & $25 \%$ & $(1291 / 5216)$ \\
\hline afectado & $88 \%$ & $(4579 / 5216)$ & $12 \%$ & $(637 / 5216)$ \\
\hline
\end{tabular}

la telicidad ejerce un leve efecto a la hora de elegir la alternativa con MDO solo en caso de que el objeto esté afectado. En cambio, la telicidad se muestra irrelevante en la respuesta de los participantes cuando se encuentran ante objetos no afectados.

\subsection{Análisis}

Los resultados del cuestionario parecen confirmar tan solo la segunda de las hipótesis formuladas en el apartado 4.2 de este capítulo (recogidas de nuevo en (91)).

(91) Hipótesis

H1: Los objetos directos de predicados télicos presentan con mayor frecuencia MDO que los objetos directos de predicados atélicos.

H2: Los objetos directos afectados por la acción del verbo presentan con mayor frecuencia MDO que los objetos no afectados. 




Gráfico 4.3: Frecuencia de respuestas atendiendo al MDO, a la afectación y a la telicidad.

Tabla 4.4: Frecuencia de respuestas atendiendo al MDO, a la afectación y a la telicidad.

\begin{tabular}{llllrl}
\hline & & \multicolumn{2}{c}{ MDO } & \multicolumn{2}{c}{ NO MDO } \\
\hline \multirow{2}{*}{ no afectado } & atélico & $75 \%$ & $(1955 / 2608)$ & $25 \%$ & $(653 / 2608)$ \\
\cline { 2 - 6 } & télico & $75 \%$ & $(1970 / 2608)$ & $25 \%$ & $(638 / 2608)$ \\
\hline afectado & atélico & $91 \%$ & $(2369 / 2608)$ & $9 \%$ & $(239 / 2608)$ \\
\cline { 2 - 6 } & télico & $85 \%$ & $(2210 / 2608)$ & $15 \%$ & $(398 / 2608)$ \\
\hline
\end{tabular}

La diferencia entre predicados télicos y atélicos no ha revelado ningún efecto claro a la hora de que los participantes se decantasen por la opción con MDO o por la opción sin marcar. No obstante, en lo que se refiere a afectación, los objetos afectados sí han demostrado presentar en mayor medida el uso de MDO, frente a los objetos no afectados, donde se ha podido apreciar que la cantidad de respuestas con marcado preposicional ha sido inferior. Por último, la observación de los resultados atendiendo a ambos factores al mismo tiempo ha reflejado una interacción entre ellos que implica la distinción de un pequeño efecto de la telicidad sobre el MDO, pero tan solo en los predicados con objetos afectados.

Estas son las conclusiones que arrojan en primera instancia los resultados, pero la metodología empleada exige un análisis más profundo de los datos. En el apartado anterior se han mostrado los resultados correspondientes a los cuatro tipos de contextos que surgen del cruce de factores planteado en la investigación; sin embargo, es necesario comprobar si todos los verbos incluidos en cada grupo se han comportado de forma más o menos homogénea. El Gráfico 4.4 refleja en un diagrama de cajas el comportamiento de los distintos grupos atendiendo a los 
porcentajes de MDO que ha recibido cada uno de los verbos que los componen. Este tipo de diagramas se utiliza para comparar la dispersión de los datos pertenecientes a distintos conjuntos. Está basado en la noción estadística de cuartill $^{22}$ y representa cada una de las cuatro partes en que los datos quedan divididos con un segmento distinto. Teniendo en cuenta que en este estudio cada conjunto de datos cuenta con ocho verbos, es decir, con ocho valores, al quedar estos divididos en cuatro partes, la caja representaría en cada caso el rango de porcentajes de MDO que han obtenido cuatro de los verbos y las líneas superior e inferior, los dos valores más altos y los dos más bajos, respectivamente.

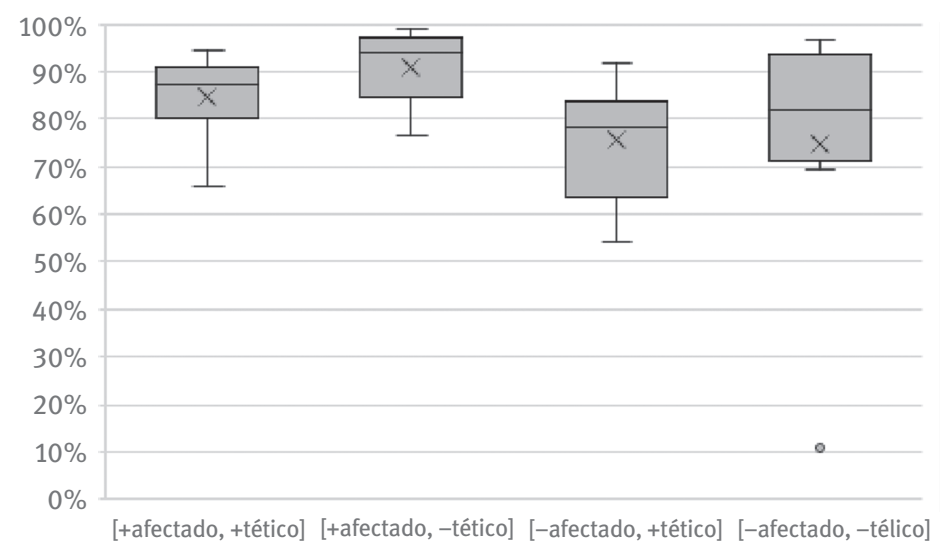

Gráfico 4.4: Porcentaje de uso del MDO con los diferentes verbos atendiendo a los distintos tipos de contexto.

Al observar el diagrama, se pueden extraer diferentes informaciones. En primer lugar, los grupos que se comportan de una manera más homogénea son aquellos cuyo objeto directo está afectado. Como se aprecia, el tamaño de las cajas es inferior en estos casos (lo que implica una menor dispersión de los datos) y, si se tienen en cuenta las tres partes superiores o, en otras palabras, seis de los ocho verbos de cada uno de esos grupos, observamos que se encuentran entre el $80 \%$ y el 94\% de casos marcados en el grupo [+afectado, +télico], y entre el 85\% y el 99\% en el grupo [+afectado, -télico]. En ambos casos, tan solo 14 puntos porcentuales explicarían la variación entre seis de los ocho verbos del grupo.

22 Cuartil: cada uno de los tres puntos que dividen un conjunto de datos numéricamente ordenados en cuatro partes iguales. A estos tres puntos se les llama primer cuartil (también llamado el cuartil inferior), segundo cuartil (el cuartil medio; es la mediana) y el tercer cuartil (cuartil superior), respectivamente. 
Por el contrario, la variación en el comportamiento de los grupos que no implican la afectación del objeto es más elevada. El grupo [-afectado, +télico] muestra la misma variación entre el comportamiento de sus verbos tanto si se escogen los tres segmentos inferiores (54-84\%), como si se observan los tres superiores (63-92\%), así pues, la diferencia para explicar el comportamiento de al menos seis de sus verbos oscilaría entre 30 y 29 puntos porcentuales, respectivamente. Finalmente, el grupo [-afectado, -télico] muestra una variación similar a la del grupo anterior, de 26 puntos si observamos los tres cuartiles superiores (71-97\%) y muchísimo más amplia si observamos los tres inferiores. En este último caso, el segmento inferior comenzaría a la altura del $11 \%$ pues la gráfica contiene lo que se conoce como un valor atípico para este grupo de verbos. Los valores atípicos son observaciones cuyo valor es muy diferente al del resto del conjunto de datos en que se inscriben. Estos distorsionan los resultados de los análisis y, por esta razón, es necesario identificarlos y, generalmente, excluirlos del análisis. En este caso, el valor atípico se corresponde con la frecuencia de uso del MDO que ha mostrado el verbo tener, cuestión que será abordada más adelante.

Por lo tanto, del Gráfico 4.4 se pueden extraer dos ideas generales. Por un lado, que la afectación se revela como un factor con mayor fuerza explicativa del MDO que la telicidad, dato que ya apuntaba también el Gráfico 4.3. Esta afirmación se desprende fundamentalmente de la comparación de los grupos en los que ambos factores actúan por separado, es decir, [+afectado, -télico] y [-afectado, +télico]. Cuando el objeto queda afectado por la acción del verbo, se observa que el comportamiento de los verbos es más homogéneo y estos tienden a aparecer con MDO con mayor frecuencia; mientras que cuando solo actúa la telicidad, la variación que adquiere la aparición del MDO es exactamente el doble de amplia y lo que ello sugiere es que el marcado proposicional lo están determinando otros factores que no han sido controlados. Por otro lado, la segunda idea indicaría la necesidad de eliminar el verbo tener del análisis y comprobar de nuevo el efecto de telicidad y afectación en el MDO. Consecuentemente, tras la eliminación de dicho verbo se han creado nuevas figuras que representan de nuevo los resultados y se ha llevado a cabo sobre estos datos un nuevo análisis estadístico.

Con respecto a la telicidad, los datos obtenidos tras la eliminación de tener aparecen recogidos en la Tabla 4.5 y representados en el Gráfico 4.5. Dichos datos indican que los participantes han elegido la opción con MDO, frente a la opción sin marcar, en un 88\% (4289/4890) de los predicados atélicos. Por otro lado, en lo referente a los predicados télicos, la opción marcada ha sido elegida en un $80 \%$ (4180/5216) de los casos. La diferencia que antes era apenas perceptible se torna mayor, alcanzando 8 puntos porcentuales, y resulta significativa en el análisis estadístico (estimado: $-0,647 ; \mathrm{p}<0,001$ ). Por lo tanto, se puede afirmar, atendiendo a los datos, que la telicidad ejerce un efecto sobre la respuesta de los 
participantes a la hora de valorar el uso del MDO. Dicho efecto consiste en que son los predicados atélicos los que parecen favorecer el uso del marcado preposicional de objeto frente a los predicados télicos, en contra de lo que se habría esperado teniendo en cuenta la mayoría de estudios previos.

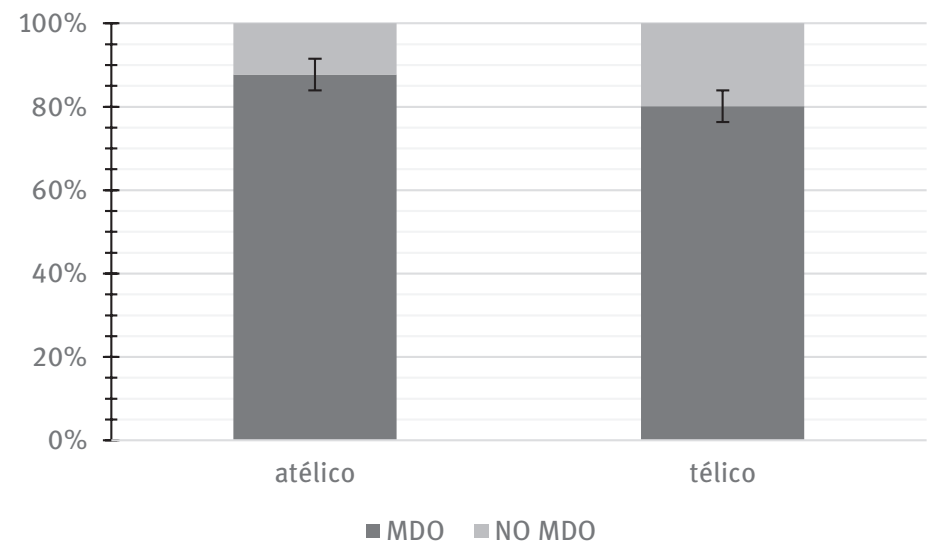

Gráfico 4.5: Frecuencia de respuestas atendiendo al MDO y a la telicidad (sin valores atípicos).

Tabla 4.5: Frecuencia de respuestas atendiendo al MDO y a la telicidad (sin valores atípicos).

\begin{tabular}{lllrr}
\hline & \multicolumn{2}{c}{ MDO } & \multicolumn{2}{c}{ NO MDO } \\
\hline atélico & $88 \%$ & $(4289 / 4890)$ & $12 \%$ & $(601 / 4890)$ \\
\hline télico & $80 \%$ & $(4180 / 5216)$ & $20 \%$ & $(1036 / 5216)$ \\
\hline
\end{tabular}

En lo que se refiere al impacto de la afectación, tras la eliminación de los resultados de tener, los datos del Gráfico 4.6 y la Tabla 4.6 muestran que los participantes han elegido la opción marcada preposicionalmente en un 79\% de los casos con objetos no afectados, frente al $88 \%$ de aquellos que contenían objetos afectados. La diferencia entre ambas categorías asciende a 9 puntos porcentuales que, respaldados nuevamente por el análisis estadístico, también suponen una diferencia significativa (estimado: 0,717; $\mathrm{p}<0,001$ ). Por lo tanto, se puede afirmar que el factor de la afectación determina de un modo significativo el hecho de que los participantes del cuestionario hayan elegido la opción con MDO en vez de la opción sin marcar.

Finalmente, en lo que a la interacción de ambos factores se refiere, los datos ya sin valores atípicos representados en el Gráfico 4.7 muestran que, en contextos con objetos no afectados, se ha elegido la alternativa con MDO en un 84\% de los 


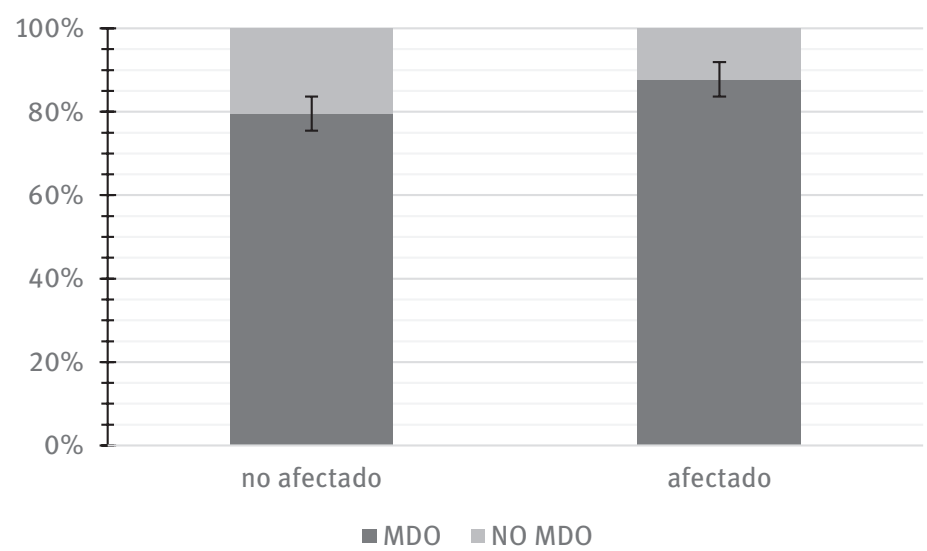

Gráfico 4.6: Frecuencia de respuestas atendiendo al MDO y a la afectación (sin valores atípicos).

Tabla 4.6: Frecuencia de respuestas atendiendo al MDO y a la afectación (sin valores atípicos).

\begin{tabular}{lccrr}
\hline & & MDO & \multicolumn{2}{c}{ NO MDO } \\
\hline no afectado & $79 \%$ & $(3890 / 4890)$ & $21 \%$ & $(1000 / 4890)$ \\
\hline afectado & $88 \%$ & $(4579 / 5216)$ & $12 \%$ & $(637 / 5216)$ \\
\hline
\end{tabular}

predicados atélicos y en un $76 \%$ de los predicados télicos; y, en lo que concierne a los objetos afectados, los participantes han elegido la opción marcada en un 91\% de los predicados atélicos, frente al 85\% de los predicados télicos. Estos resultados, cuyos valores absolutos aparecen recogidos en la Tabla 4.7, excluyen la interacción propuesta en la primera revisión de los datos, pues el leve efecto que se atestigua de la telicidad se produciría de forma independiente al impacto de la afectación. La ausencia de interacción queda, asimismo, avalada por el análisis estadístico (estimado: -0,01548; $\mathrm{p}=0,898$ ).

Los resultados de este capítulo, por tanto, han confirmado un efecto claro por parte de la afectación del objeto, mientras que han apuntado hacia un leve efecto de la telicidad sobre el marcado preposicional, que parece apuntar en la dirección opuesta a la esperada. No obstante, es necesario mantener una posición cauta acerca de este efecto por la forma en que se ha manifestado, puesto que, aunque al final se ha revelado como significativo, se ha demostrado que los dos conjuntos de contextos en los que no actuaba la afectación mostraban gran dispersión en los resultados, por lo que es probable que puedan haber intervenido otros factores que no hayan sido controlados, tales como la preferencia por la selección de objetos con referente humano o la agentividad potencial del objeto. 


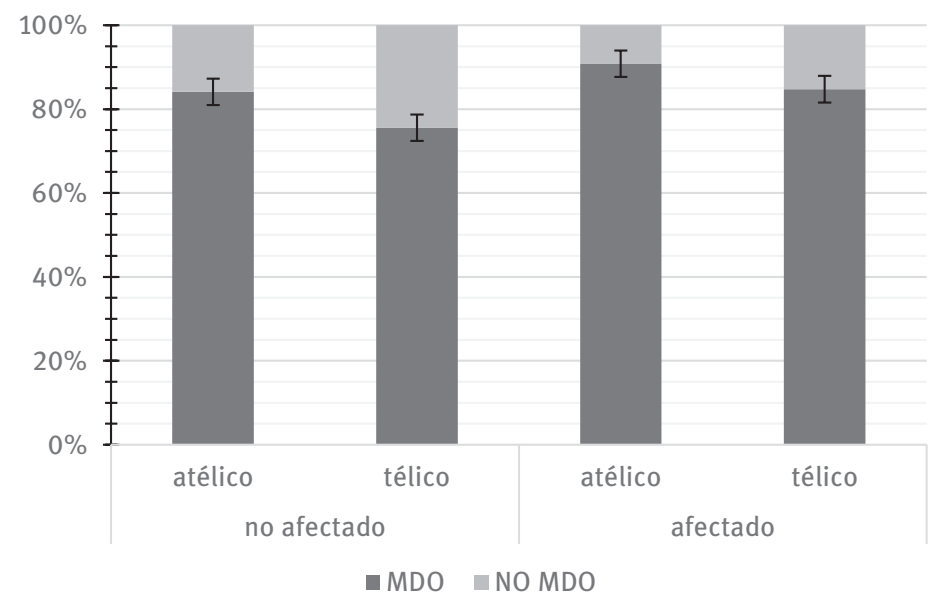

Gráfico 4.7: Frecuencia de respuestas atendiendo al MDO, a afectación y a telicidad (sin valores atípicos).

Tabla 4.7: Frecuencia de respuestas atendiendo al MDO, a afectación y a telicidad (sin valores atípicos).

\begin{tabular}{llllrl}
\hline & & \multicolumn{2}{c}{ MDO } & \multicolumn{2}{c}{ NO MDO } \\
\hline \multirow{2}{*}{ no afectado } & atélico & $84 \%$ & $(1920 / 2282)$ & $16 \%$ & $(653 / 2282)$ \\
\cline { 2 - 6 } & télico & $76 \%$ & $(1970 / 2608)$ & $24 \%$ & $(638 / 2608)$ \\
\hline \multirow{2}{*}{ afectado } & atélico & $91 \%$ & $(2369 / 2608)$ & $9 \%$ & $(239 / 2608)$ \\
\cline { 2 - 6 } & télico & $85 \%$ & $(2210 / 2608)$ & $15 \%$ & $(398 / 2608)$ \\
\hline
\end{tabular}

Por lo tanto, es conveniente continuar analizando algunos de los verbos empleados con más detalle.

En primer lugar, es interesante ver qué ha ocurrido con el verbo tener. La pregunta de la que formaba parte es la que aparece reflejada en (92) y los participantes seleccionaron la opción de objeto directo marcado tan solo en el 11\% (35/326) de los casos.

(92) Pregunta del cuestionario con el verbo tener

Pablo tuvo [un ayudante/a un ayudante] durante varios años.

Algunos autores han señalado que no es normal que este verbo reciba marcado preposicional si el objeto no presenta ningún tipo de predicación secundaria y que, en el caso de aparecer la marca preposicional, esta implicaría un cambio de significado respecto a la relación que se establece entre sujeto y objeto (Laca 1987, 298; Torrego 1999, 1793; Fábregas 2013, 24). De hecho, ha habido autores que 
han llegado a postular una restricción de definitud en el objeto directo del verbo tener, similar a la que se produce en la posición posverbal de las construcciones existenciales (Partee 1999; Gutiérrez-Rexach 2000). Por lo tanto, el problema en el caso del cuestionario parece derivarse de su aparición sin ningún tipo de predicación secundaria, pero esta no se incluyó porque una premisa de la metodología era mantener la misma estructura en todas las preguntas y la inclusión de predicación secundaria en los objetos hubiese provocado el aumento del MDO, pues, como han apuntado diversos estudios, la predicación secundaria es un factor que favorece la aparición del fenómeno (Laca 1987; Torrego 1999; Ormazábal/Romero 2007; López 2012); y, por consiguiente, los resultados hubiesen quedado «contaminados» por el efecto de otro factor que no formaba parte del estudio.

En lo que respecta al leve efecto que los verbos atélicos parecen ejercer sobre el MDO favoreciendo su uso, la explicación es probable que resida en el comportamiento aislado de determinados verbos. No es sorprendente, por ejemplo, que los verbos amar y admirar hayan obtenido porcentajes de marcado del $97 \%$ (315/326) y del 88\% (286/326), respectivamente. Estos verbos se incluyeron en el conjunto [-télico, -afectado] porque ambos cumplían los requisitos semánticos de dar pie a predicados atélicos y no implicar un objeto directo afectado. El objetivo era comprobar si su comportamiento se ajustaba al del resto del conjunto o si, por el contrario, los resultados apuntaban hacia donde han señalado otros estudios con relación a estos verbos. Laca $(2006,451)$ sostiene que tanto los verbos estativos que implican relaciones espaciales, temporales o de grado entre sujeto y objeto, como aquellos que expresan actitudes afectivas (como es el caso de amar o admirar) han exigido MDO desde los primeros textos que su estudio tiene en cuenta, y estos datan del siglo XII. Quizá esta exigencia no sea tal y como la plantea Laca, en ese sentido de obligatoriedad desde el comienzo, puesto que, como se verá en el siguiente capítulo, en la búsqueda de corpus, el verbo amar con objetos directos indefinidos parte con una frecuencia relativa del 50\% (4/8) de casos marcados en el siglo XIV; pero es evidente que ya desde el comienzo de expansión del fenómeno, estos verbos manifestaban una clara preferencia por la marcación de su objeto.

En el mismo grupo de verbos que amar y admirar, se encuentra el verbo oír. Asimismo, dicho verbo ha obtenido una frecuencia muy elevada de respuesta con MDO (87\% (284/326)), a pesar de pertenecer al grupo de contextos que implican la atelicidad del predicado y la ausencia de afectación en el objeto. Los verbos de percepción se caracterizan por presentar estructuras argumentales con un nivel de transitividad muy reducido puesto que la transferencia de energía no se produce desde un sujeto agente a un objeto paciente, sino que un sujeto experimentante percibe un estímulo que funciona como fuente o causa del propio evento. Sin embargo, Enghels (2012) defiende que dentro del propio grupo de los verbos de per- 
cepción se puede establecer una gradación con respecto al nivel de transitividad que presentan, atendiendo a las propiedades de agentividad que se le atribuyen al estímulo de los diferentes verbos. De esta manera, al estímulo del verbo oír se le presupone un cierto grado de agentividad, pues ha de tratarse de un objeto que al menos tenga la capacidad de producir un ruido o un sonido, como se puede observar en (93), frente a estímulos como los del verbo ver, a los que solo es necesario presuponerles la existencia.

(93) Verbos de percepción

a. Veo la casa.

b. \#Oigo la casa.

Según Enghels (2012, 45), el objeto directo de oír es, por tanto, más susceptible de ser marcado con la preposición a que el objeto de ver, puesto que se aleja en mayor medida de las características prototípicas de los pacientes. La elevada frecuencia de marcado del objeto de oír queda respaldada también por los datos de la búsqueda de corpus presentada en el capítulo siguiente, donde dicho verbo parte con una frecuencia de aparición del MDO de un 60\% (12/20) ya en el siglo XIV.

En definitiva, la disparidad tan grande encontrada en el comportamiento de los verbos del grupo [-afectado, -télico], como se ha podido observar en la explicación particular de determinados casos, en realidad es debida a la acción de otros factores que no se han controlado en el cuestionario, tales como la agentividad del objeto o la existencia de predicación secundaria. Este hecho no es sorprendente pues el grupo mencionado está definido por la ausencia de los dos factores investigados y, por lo tanto, la explicación del MDO en este caso ha de fundamentarse en factores que no hayan sido contemplados en el diseño del experimento. No obstante, metodológicamente era necesaria la introducción de dicho grupo para descartar que los factores investigados no fuesen determinantes en el fenómeno hasta el punto de que su ausencia bloquease la aparición del marcado.

\subsection{Conclusiones}

La investigación sincrónica llevada a cabo a través del cuestionario gramatical de elección forzada ha arrojado unos resultados que apuntan a que tanto la afectación como la telicidad parecen ejercer un impacto relevante sobre el MDO en español; sin embargo, mientras que la afectación desempeña un efecto más contundente en el sentido marcado por la hipótesis de partida, el impacto de la teli- 
cidad se revela como una influencia más leve que, además, apunta en dirección opuesta a lo que había propuesto la bibliografía anterior.

La afectación se postula como un factor sin duda relevante para la explicación del MDO en español. Siguiendo la línea marcada por diversos estudios previos (Fernández Ramírez 1951; Pottier 1968; Torrego 1999; 2002; von Heusinger/Kaiser 2011), el marcado preposicional es un fenómeno relacionado en cierta medida con aquellos objetos directos cuya afectación está implicada en algún grado por la semántica del verbo al que acompañan. Dicha relación se ha comprobado desde un punto de vista sincrónico y, como se decía en el capítulo 1, una vez comprobado el efecto del factor, ahora cabe preguntarse si este ha tenido lugar asimismo en etapas previas de la evolución del MDO.

Por otro lado, la telicidad ha mostrado un efecto que, a pesar de no ser demasiado fuerte, apunta a que son los predicados atélicos aquellos que parecen favorecer el marcado o, al menos, ser más propicios para la aparición de la marca preposicional. Este resultado puede, a primera vista, no estar exento de polémica puesto que señala en la dirección opuesta a la que habían apuntado los estudios previos acerca del tema (Torrego 1999; 2002) y, en algunos casos, aparece solapado con los efectos de otros factores cuya influencia también se ha postulado para la explicación del MDO. Sin embargo, podría encontrar una explicación en la línea de aquellos estudios que proponen que el marcado preposicional está relacionado no solo con grados extremadamente altos de transitividad, sino también con grados extremadamente bajos (García García 2014; 2018). Esta idea ofrece una explicación al marcado obligatorio de aquellos objetos inanimados, agentivos en cierta medida, que aparecen con verbos estativos que implican relaciones espaciales, temporales o de grado entre sujeto y objeto.

Sea como fuere, el objetivo de este capítulo ha sido el de buscar respuesta a las dos preguntas principales que se planteaban en los objetivos de la introducción:

- ¿Influye la telicidad del predicado en el uso del MDO?

- ¿Contribuye la afectación del objeto a la aparición de la marca preposicional?

Puesto que la respuesta a ambas ha sido afirmativa (teniendo en cuenta los matices para cada una que se han descrito antes), el capítulo 5 se ocupa, por tanto, de intentar dar respuesta a la primera de las preguntas más específicas que se planteaban siempre y cuando las primeras obtuviesen una respuesta afirmativa:

- ¿El efecto de la telicidad/afectación se puede apreciar tan solo a nivel sincrónico o es posible documentarlo, asimismo, a nivel diacrónico? 
Así pues, en el siguiente capítulo se presenta un estudio que ha consistido en el análisis de un corpus diacrónico y cuyo objetivo es comprobar de qué manera explican los datos diacrónicos los efectos hallados en la investigación sincrónica y hasta qué punto los factores estudiados han contribuido a la expansión del MDO en la historia del español. Los resultados obtenidos en dicho análisis han mostrado una importante coherencia con los presentados en este capítulo, señalando la importancia de la afectación y ayudando a esclarecer qué es lo que ocurre con la telicidad. 
